Arch Virol (1994) [Suppl] 9: 451-459

Archives

Virrology

(C) Springer-Verlag 1994

\title{
Recognition of cellular receptors by bovine coronavirus
}

\author{
B. Schultze and G. Herrler \\ Institut für Virologie, Philipps-Universität Marburg, Marburg, \\ Federal Republic of Germany
}

Summary. Bovine coronavirus (BCV) initiates infection by attachment to cell surface receptors the crucial component of which is $\mathrm{N}$-acetyl-9-Oacetylneuraminic acid. Inactivation of receptors by neuraminidase treatment and restoration of receptors by enzymatic resialylation of asialo-cells is described as a method to determine (i) the type of sialic acid that is recognized; (ii) the linkage specificity of the viral binding activity; (iii) the minimal amount of sialic acid required for virus attachment. Evidence is presented that both glycoproteins and glycolipids can serve as receptors for $\mathrm{BCV}$ provided they contain 9-O-acetylated sialic acid. A model is introduced proposing that after initial binding to sialic acid-containing receptors, the S-protein of BCV interacts with a specific protein receptor. This interaction may result in a conformational change that exposes a fusogenic domain and thus induces the fusion between the viral and the cellular membrane.

\section{Introduction}

The interactions between viruses and cell surface receptors are of major interest because they often determine the cell tropism of the respective virus. In addition they are a promising target for antiviral chemotherapy. Recently progress was made in elucidating the receptors for several coronaviruses. The picture emerging from the data reported appears rather complex. A member of the carcinoembryonic antigen family of proteins has been shown to serve as a receptor for mouse hepatitis virus (MHV) strain A59 [1]. Transmissible gastroenteritis virus (TGEV) and human coronavirus, strain $229 \mathrm{E}$ (HCV-229E), have been reported to use aminopeptidase $\mathrm{N}$ as a receptor for the infection of cells [2,3]. Another variation in the recognition of receptors has been reported for bovine coronavirus $(\mathrm{BCV})$. The strategy this virus uses to attach to the cell surface is similar to that of influenza $\mathrm{C}$ virus, i.e. the major receptordeterminant recognized is $\mathrm{N}$-acetyl-9-O-acetylneuraminic acid (Fig. 1) 


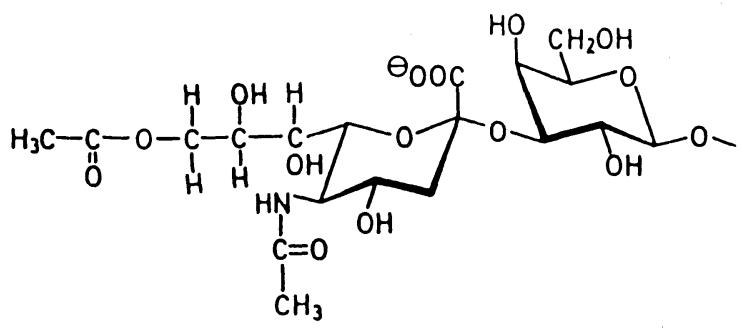

Fig. 1. Structure of N-acetyl-9-O-acetylneuraminic acid, the receptor determinant for $\mathrm{BCV}$. The sugar is attached in an $\alpha 2,3$-linkage to galactose. Another common linkage type is the $\alpha 2,6$-linkage

[4-7]. This sugar is required on the cell surface not only to bind to erythrocytes in the hemagglutination reaction, but also to attach to and subsequently infect cultured cells $[8,9]$.

The importance of CEA and aminopeptidase as virus receptors has been established by two experimental techniques: (i) Monoclonal antibodies directed against these surface proteins are able to prevent virus infection. (ii) Receptor-negative cells become susceptible to infection after expression of the corresponding gene from permissive cells. These approaches are not applicable to viruses that use a sugar as a receptor determinant, as is the case with $\mathrm{BCV}$.

\section{Analysis of sialic acid as receptor determinant for viruses}

Sialic acid is recognized as a receptor determinant by more viruses than any other determinant known. Both enveloped viruses (influenza viruses, some paramyxoviruses, some coronaviruses) and non-enveloped viruses (reoviruses, encephalomyocarditis virus, polyomavirus) have been reported to attach to glycoconjugates containing this type of sugar [10]. In order to determine the importance of sialic acid for binding of virus to cells, the approach shown in Fig. 2 has been used with success. Cells may contain different types of sialic acid, i.e. derivatives of neuraminic acid with different substitutents at the amino group ( $\mathrm{N}$-acetyl or $\mathrm{N}$-glycolylneuraminic acid) or the various hydroxyl groups, e.g. 9-Oacetyl-N-acetylneuraminic acid. The sialic acid molecules may be attached to the glycoconjugates in different linkages, e.g. $\alpha 2,3$ or $\alpha 2,6$-linked to galactose (Fig. 1). Incubation of cells with neuraminidase results in the release of surface-bound sialic acid (Fig. 2). These asialo-cells are expected to be resistant to infection, if a virus requires sialic acid for attachment to cells. However, resistance to infection can also be due to a nonspecific effect, e.g. due to a change in the nagative charge of the cell surface. Such effects can be ruled out by showing that the virus specifically recognizes a certain type of sialic acid or a certain linkage type. For 

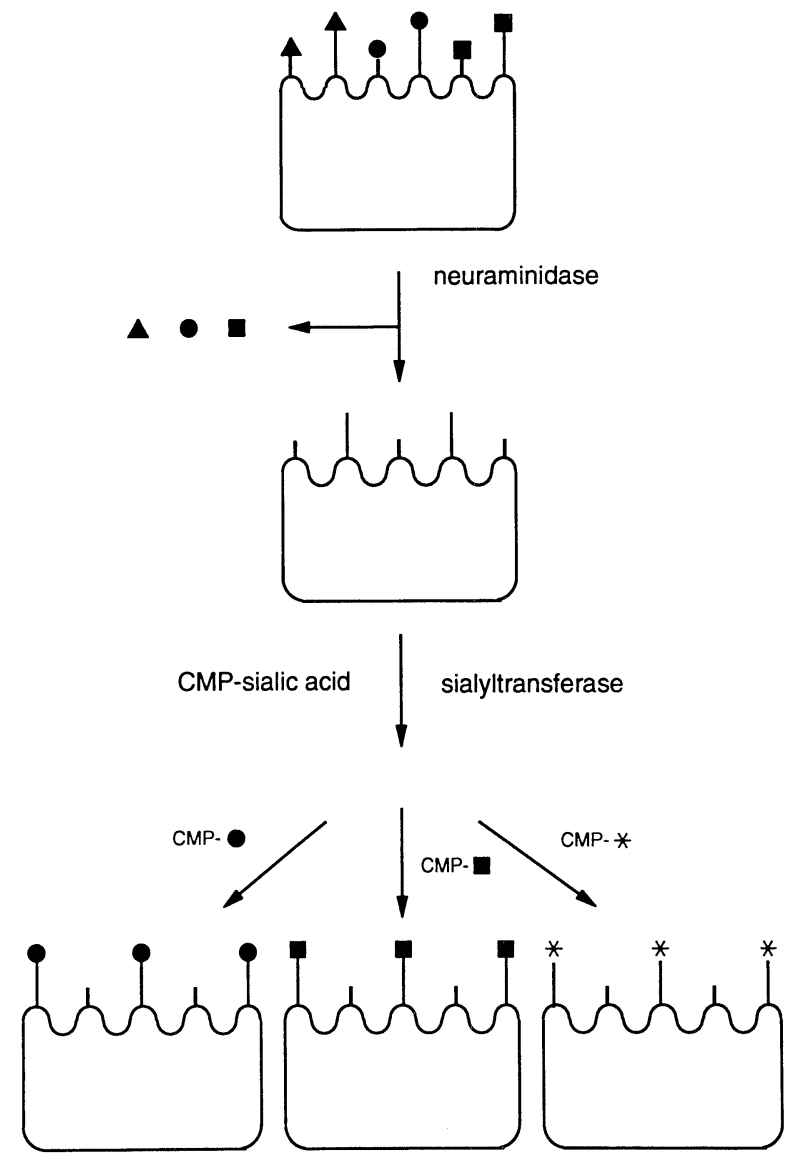

Fig. 2. Schematic presentation of the resialylation method. Sialic acid is released from the cell surface by treatment with neuraminidase. Asialo cells are resialylated by incubation with sialyltransferase and CMP-activated sialic acids. Because of the specificity of the enzyme and the choice of sialic acid, cells are obtained which contain only one type of sialic acid in a defined linkage type on the surface. Squares, circles, triangles, and asterisks represent different types of sialic acid. Long and short bars represent different linkage types, e.g. $\alpha 2,3$ and $\alpha 2,6$-linkage to galactose

this purpose, asialo-cells are incubated with sialyltransferase and CMPactivated sialic acid. Because of the specificity of the enzyme, sialic acids are attached to cell surface glycoconjugates in a defined linkage, e.g. $\alpha 2,6$-linked to galactose. The choice of CMP-activated sialic acid determines what type of sialic acid is transferred to the cell surface. In this way it is possible to obtain cells that contain only a single type of sialic acid attached in a single type of linkage (Fig. 2). The resialylated cells are tested either for agglutination by virus, in the case of erythrocytes, or for their susceptibility to infection, in the case of cultured cells. 


\section{Importance of 9-0-acetylated sialic acid as a receptor determinant for bovine coronavirus}

Using the approach described above, it has been shown that BCV uses $\mathrm{N}$-acetyl-9-O-acetylneuraminic acid as a receptor determinant not only for agglutination of erythrocytes, but also for infection of cultured cells $[5,8]$. Sialic acid lacking a 9-O-acetyl group is not effective in this respect. Studies with erythrocytes indicate that hemagglutinating encephalomyelitis virus (HEV) and human coronavirus OC43 also require $\mathrm{Neu} 5,9 \mathrm{Ac}_{2}$ for attachment to cells $[4,5]$. The resialylation method can be applied to compare the efficiency of two viruses in recognizing the same receptor determinant. For this purpose, aliquots of cells are resialylated using limiting concentrations of the activated sialic acid. This produces cells that have different amounts of sialic acid and, therefore, different numbers of receptors on their surface. As shown in Table 1, influenza $\mathrm{C}$ virus is more capable than is $\mathrm{BCV}$ in recognizing Neu5,9 $\mathrm{Ac}_{2}$ present in an $\alpha 2,6$-linkage; alternatively, if the same type of sugar is present in an $\alpha 2,3$-linkage, BCV requires a lower amount of sialic acid than does influenza $\mathrm{C}$ virus for agglutination of erythrocytes. Thus, the two viruses recognize $\mathrm{Neu} 5,9 \mathrm{Ac}_{2}$ in both linkage types but with different efficiencies.

\section{Glycoproteins and glycolipids as receptors for BCV}

As sialic acid is present on both glycoproteins and glycolipids, the question arises whether both types of glycoconjugates can serve as receptors for BCV. The sialyltransferase used for successful restoration of receptors on MDCK I cells was specific for the $\alpha 2,6$-linkage [8]. As this linkage is typically found on oligosaccharides of glycoproteins but not on gangliosides, the receptors generated by $\alpha 2,6$ sialyltransferase are glycoproteins. Thus, glycoproteins containing $\mathrm{Neu} 5,9 \mathrm{Ac}_{2}$ can serve as receptors for $\mathrm{BCV}$. Hemagglutination-inhibition studies indicated that not only glycoproteins but also gangliosides can prevent $\mathrm{BCV}$ from agglutinating red blood cells. Bovine brain gangliosides are known to contain $\mathrm{Neu} 5,9 \mathrm{Ac}_{2}$ and, therefore, were analyzed for their ability to serve as virus receptors. If cells are incubated with gangliosides, the glycolipids attach to the cell surface via ionic interactions. Part of the bound gangliosides are incorporated into the lipid bilayer of the plasma membrane. As shown in Table 2, bovine brain gangliosides restored the susceptibility of neuraminidase-treated cells to infection to the same extent as resialylation. This result indicates that BCV can use both glycoproteins and glycolipids as receptors, provided they contain $\mathrm{Neu} 5,9 \mathrm{Ac}_{2}$. 
Table 1. Comparison of the ability of bovine coronavirus (BVC) and influenza $\mathrm{C}$ virus (JHB/1/66) to agglutinate erythrocytes containing $\mathrm{N}$-acetyl-9-O-acetylneuraminic acid in either of two different linkage types

\begin{tabular}{|c|c|c|}
\hline \multirow[b]{2}{*}{ Erythrocytes } & \multicolumn{2}{|c|}{ Hemagglutination titer (units/ml) } \\
\hline & $\mathrm{BCV}$ & $\mathrm{C} / \mathrm{JHB} / 1 / 66$ \\
\hline Asialo & $<2$ & $<2$ \\
\hline \multicolumn{3}{|l|}{ Resialylated } \\
\hline \multicolumn{3}{|l|}{ Neu5Aca2,3Gal $\beta 1,4$ GlcNAc } \\
\hline CMP-Neu5Ac, $0.50 \mathrm{nmol}$ & 64 & $<2$ \\
\hline $1.00 \mathrm{nmol}$ & 256 & $<2$ \\
\hline $2.00 \mathrm{nmol}$ & 2048 & 128 \\
\hline \multicolumn{3}{|l|}{ Neu5Ac $2,6 \mathrm{Gal} \beta 1,4 \mathrm{GlcNAc}$} \\
\hline CMP-Neu5Ac, $0.25 \mathrm{nmol}$ & $<2$ & 512 \\
\hline $0.50 \mathrm{nmol}$ & $<2$ & 512 \\
\hline $1.00 \mathrm{nmol}$ & $<2$ & 512 \\
\hline $2.00 \mathrm{nmol}$ & $<2$ & 512 \\
\hline $4.00 \mathrm{nmol}$ & 128 & 512 \\
\hline
\end{tabular}

Table 2. Restoration of receptors for bovine coronavirus on neuraminidase-treated MDCK I cells by resialylation or by incubation with bovine brain gangliosides

\begin{tabular}{ll}
\hline Treatment & $\begin{array}{l}\text { Hemagglutination titer } \\
\text { (units/ml) of the supernatant }\end{array}$ \\
\hline None & 256 \\
Neuraminidase & $<2$ \\
Neuraminidase, resialylated & 32 \\
Neuraminidase, gangliosides & 32 \\
\hline
\end{tabular}

The $\mathbf{S}$ protein is the receptor-binding protein of coronaviruses

Differences among coronaviruses have been reported not only with respect to their cellular receptors but also with respect to their viral receptor-binding proteins [11]. In the case of infectious bronchitis virus (IBV), transmissible gastroenteritis virus (TGEV), and related viruses, the $\mathrm{S}$ protein is responsible for attachment to cells. $\mathrm{BCV}$ and the serologically related viruses $\mathrm{HEV}$ and $\mathrm{HCV}-\mathrm{OC} 43$ contain an $\mathrm{HE}$ protein missing from TGEV, IBV, and related viruses. The HE protein of $\mathrm{BCV}$ has acetylesterase activity $[12,13]$. In addition, it has been suggested to function as a hemagglutinin, i.e. to be responsible for attachment to 
erythrocytes [14]. Surprisingly, the S protein of BCV was found recently to have hemagglutinating activity, too. Both proteins use Neu5,9 $\mathrm{Ac}_{2}$ as a receptor-determinant for attachment to cells [8]. However, the $\mathrm{S}$ protein is a more efficient hemagglutinin than is $\mathrm{HE}$, because it requires less 9-O-acetylated sialic acid, i.e. fewer receptor determinants, on the cell surface for the agglutination of erythrocytes [8]. Therefore, the $\mathrm{S}$ protein is suggested to be the major receptor-binding protein of all coronaviruses.

\section{Is one virus receptor sufficient for infection?}

As described in the introductory section, different receptors have been described for different coronaviruses, a sugar as receptor determinant for $\mathrm{BCV}$, and specific proteins as receptors for MHV, TGEV, and HCV$229 \mathrm{E}$. It may appear surprising that coronaviruses apply such different strategies for binding to the cell surface. However, initiation of an infection is a complex process that comprises both attachment to the cell surface and, in the case of enveloped viruses, fusion between the membranes of the virus and the target cell. For some viruses a single type of receptor may be sufficient to induce both events. The hemagglutinin of influenza viruses, for example, binds to sialic acid-containing receptors and causes fusion after a conformational change which exposes a fusogenic epitope. The change in the conformation is triggered by the acid $\mathrm{pH}$ of the endosome after endocytotic uptake of the virus. However, the fusion activity of many viruses, including coronaviruses, is acid-independent. For these viruses fusion must be activated by factors other than $\mathrm{pH}$.

The interaction of the viral fusion protein with a cell surface protein might be such a factor, as shown by our model of the inital events of an infection by BCV (Fig. 3). The first contact between the virus and the

cell is established by binding of the $\mathrm{S}$ protein to 9-O-acetylated sialic acid (Fig. 3A). The initial attachment sites are most likely glycoproteins. On cells that lack appropriate glycoproteins, gangliosides containing $\mathrm{Neu} 5,9 \mathrm{Ac}_{2}$ can serve as receptors as well. In fact, it may be favorable for the virus to proceed from the glycoprotein receptor to glycoplipids in order to obtain closer contact between the viral and cellular lipid bilayer. The receptor-destroying enzyme, i.e. the acetylesterase of $\mathrm{BCV}$, might be helpful in accomplishing this transition. Binding to sialic acid-containing receptors appears not to be sufficient for $\mathrm{BCV}$ to trigger the fusion event, because BCV does not cause hemolysis of erythrocytes, despite efficient agglutination. Therefore, we assume that the binding of the $\mathrm{S}$ protein to a second type of receptor (Fig. 3B) is required to induce a conformational change, one which results in exposure of a fusiogenic 


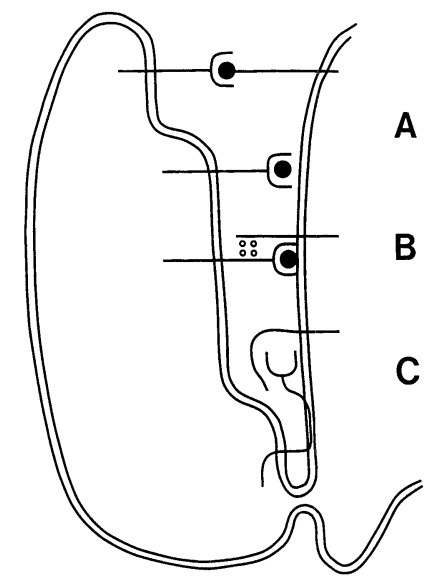

Fig. 3. Model of the initial events of an infection by BCV. Virus attaches to the cell surface by binding of the $\mathrm{S}$ protein to 9-O-acetylated sialic acid (solid circle) of glycoproteins or glycolipids $(A)$. Interaction with a specific protein, which serves as a second receptor $(B)$, results in a conformational change and exposes a fusogenic epitope of the $\mathrm{S}$ protein. This epitope interacts with the plasma membrane $(C)$ and induces the fusion of the viral envelope with the cellular membrane

epitope (Fig. 3C). Interaction of the fusion-active S-protein with the target membrane destabilizes lipid bilayers and allows fusion between the virus and the cellular membrane.

The existence of a second receptor for $\mathrm{BCV}$ remains to be proven. It is interesting to note that coronaviruses, which recognize specific proteins as receptors, also have a sialic acid-binding activity. TGEV has been shown recently to agglutinate erythrocytes after pretreatment of the virus with neuraminidase to release inhibitory compounds from the viral surface [16]. As has been reported for infectious bronchitis virus, sialic acid $\alpha 2,3$-linked to cell surface compounds serves as a receptor determinant for TGEV on erythrocytes $[16,17]$. Some murine coronaviruses are able to agglutinate mouse erythrocytes, suggesting that they recognize 9-O-acetylated sialic acid [18]. Thus, the ability to bind to two types of receptors is not restricted to $\mathrm{BCV}$. However, the importance of the sugar-binding activity may vary among coronaviruses. Whereas $\mathrm{BCV}$ requires 9-O-acetylated sialic acid for efficient attachment to cultured cells, other viruses, such as MHV, may have optimized the recognition of the protein receptor in such a way that they are no longer dependent on the recognition of a carbohydrate receptor determinant.

\section{Acknowledgements}

We are grateful to Dr. Dr. R. Brossmer for providing CMP-Neu5,9Ac 2 . This work was supported by grants from Deutsche Forschungsgemeinschaft (He 1168/2-1,2). 


\section{References}

1. Dveksler GS, Pensiero MN, Cardelichio CB, Williams RK, Jiang G-S, Holmes KV, Dieffenbach CW (1991) Cloning of the mouse hepatitis virus (MHV) receptor: expression in human and hamster cell lines confers susceptibility. J Virol 65: $6881-6891$

2. Delmas B, Gelfi J, L'Haridon R, Vogel LK, Sjöström H, Noren O, Laude H (1992) Aminopeptidase $\mathrm{N}$ is a major receptor for the enteropathogenic coronavirus TGEV. Nature 357: 417-420

3. Yeager CL, Ashmun RA, Williams RK, Cardellichio CB, Shapiro LH, Look AT, Holmes KV (1992) Human aminopeptidase $\mathrm{N}$ is a receptor for human coronavirus 229E. Nature 357: 420-422

4. Vlasak R, Luytjes W, Spaan W, Palese P (1988) Human and bovine coronaviruses recognize sialic acid containing receptors similar to those of influenza $C$ virus. Proc Natl Acad Sci USA 85: 4526-4529

5. Schultze B, Gross H-J, Brossmer R, Klenk H-D, Herrler G (1990) Hemagglutinating encephalomyelitis virus attaches to $\mathrm{N}$-acetyl-9-O-acetylneuraminic acidcontaining receptors on erythrocytes: comparison with bovine coronavirus and influenza C virus. Virus Res 16: 185-194

6. Herrler G, Rott R, Klenk H-D, Müller H-P, Shukla AK, Schauer R (1985) The receptor-destroying enzyme of influenza $\mathrm{C}$ virus is neuraminate-O-acetylesterase. EMBO J 4: 1503-1506

7. Rogers GN, Herrler G, Paulson JC, Klenk H-D (1986) Influenza C virus uses 9-Oacetyl-N-acetylneuraminic acid as a high affinity receptor determinant for attachment to cells. J Biol Chem 261: 5947-5951

8. Schultze B, Herrler G (1991) Bovine coronavirus uses N-acetyl-9-O-acetylneuraminic acid as a receptor determinant to initiate the infection of cultured cells. J Gen Virol 73: 901-906

9. Herrler G, Klenk H-D (1987) The surface receptor is a major determinant of the cell tropism of influenza C virus. Virology 159: 102-108

10. Lentz TL (1990) The recognition event between virus and host cell receptor: a target for antiviral agents. J Gen Virol 71: 751-766

11. Spaan W, Cavanagh D, Horzinek MC (1988) Coronaviruses: structure and genome expression. J Gen Virol 69: 2939-2952

12. Vlasak R, Luytjes W, Leider J, Spaan W, Palese P (1988) The E3 protein of bovine coronavirus is a receptor-destroying enzyme with acetylesterase activity. $\mathrm{J}$ Virol 62: 4686-4690

13. Schultze B, Wahn K, Klenk H-D, Herrler G (1991) Isolated HE protein from hemagglutinating encephalomyelitis virus and bovine coronavirus has receptordestroying and receptor-binding activity. Virology 180: 221-228

14. King B, Potts BJ, Brian DA (1985) Bovine coronavirus hemagglutinin protein. Virus Res 2: 1010-1013

15. Schultze B, Gross H-J, Brossmer R, Herrler G (1991) The S protein of bovine coronavirus is a hemagglutinin recognizing 9-O-acetylated sialic acid as a receptor determinant. J Virol 65: 6232-6237

16. Schultze B, Enjuanes L, Cavanagh D, Herrler G (1993) N-acetylneuraminic acid plays a critical role for the haemagglutinating activity of avian infectious bronchitis virus and porcine transmissible gastroenteritis virus. In: Laude $\mathrm{H}$, Vautherot JF (eds) Coronaviruses: molecular biology and pathogenesis (in press) 
17. Schultze B, Cavanagh D, Herrler G (1992) Neuraminidase treatment of avian infectious bronchitis coronavirus reveals a hemagglutinating activity that is dependent on sialic acid-containing receptors on erythrocytes. Virology 189: 792-794

18. Sugiyama K, Amano Y (1980) Hemagglutination and structural polypeptides of a new coronavirus associated with diarrhea in infant mice. Arch Virol 66: 95-105

Authors' address: Dr. G. Herrler, Institut für Virologie, Philipps-Universität, Biegenstrasse 10, D-35037 Marburg, Federal Republic of Germany. 